\title{
LEUCEMIA MIELOIDE EXTRAMEDULAR CON COMPROMISO ENDOMETRIAL: REPORTE DE UN CASO Y REVISIÓN DE LA LITERATURA
}

\section{Extramedullary myelogenous leukemia with endometrial involvement: a case report and literature review}

Germán García-Soto, M.D.*, Juan de Jesús Pachón-Rincón, M.D.**, Jorge A. Castaño, M.D.***, José Domingo Torres, M.D.****, Elvia Inés Gómez, M.D. *****

\section{RESUMEN}

La leucemia mieloide extramedular (LME), también conocida como sarcoma granulocítico, es una manifestación extramedular de la leucemia mieloide aguda. Puede presentarse virtualmente en todos los sitios del organismo y en cualquier momento de la evolución de la enfermedad primaria. Se define como una lesión tumoral compuesta de células mieloides inmaduras que se localiza en cualquier sitio extramedular.

Se reporta el caso de una paciente de 39 años de edad quien se presentó con compromiso uterino que simuló un carcinoma endometrial y en quien finalmente se diagnosticó LME con compromiso uterino, en el contexto de una leucemia mieloide aguda (LMA). Se presenta una revisión de la literatura y se resaltan los aspectos más importantes de la enfermedad.

Metodología: con el fin de conocer los aspectos más relevantes de esta enfermedad como frecuencia,

* Ginecólogo Oncólogo. Profesor del Grupo de Ginecología Oncológica. Universidad de Antioquia. Medellín, Colombia. Correo electrónico: gegar65@yahoo.com

** Especialista en Ginecología y Obstetricia. Universidad de Antioquia.

*** Patólogo. Profesor del Departamento de Patología. Universidad de Antioquia.

**** Hematólogo Internista. Profesor del Departamento de Medicina interna Universidad de Antioquia.

***** Patóloga. Hospital Universitario San Vicente de Paúl, Medellín, Colombia. manifestaciones clínicas y diagnóstico histopatológico, tratamiento y pronóstico, reportados en la literatura, se utilizaron las bases de datos Medline y Ovid y se hizo la búsqueda con las palabras clave granulocytic sarcoma, extramedullary leukemia, myeloid sarcoma, female genital tract, uterus, uterine cervix, endometrium.

Resultados: dos revisores evaluaron la pertinencia de los artículos y seleccionaron aquellos que trataban los aspectos relevantes del tema o hacían referencia al tracto genital femenino y específicamente al útero (uterus, uterine cervix). Se realizó nueva búsqueda con base en las referencias de algunos artículos. La búsqueda arrojó en total 123 artículos que se referían a diversos aspectos de la enfermedad y las diferentes partes del cuerpo involucradas. Se seleccionaron 14 artículos pertinentes, incluyendo uno de revisión sobre leucemia mieloide extramedular en general. La mayoría de los artículos son reporte de casos, reportes breves y revisión de algunos aspectos de la enfermedad, en series pequeñas de pacientes. Ningún artículo se refiere específicamente al compromiso del endometrio.

Conclusiones: el compromiso del útero por LME es raro y la información disponible en la literatura acerca de esta entidad es escasa, y consiste básicamente de informes de casos y de análisis por agrupamiento de los temas publicados. 
Hay consenso en la dificultad del diagnóstico inicial de esta enfermedad, en su mal pronóstico y en su tratamiento, aunque la experiencia es limitada.

Palabras clave: leucemia mieloide aguda, leucemia mieloide extramedular, sarcoma granulocítico, tracto genital femenino, útero, endometrio.

\section{SUMMARY}

Extramedullary myelogenous leukemia (also known as granulocytic sarcoma) is an extramedullary manifestation of acute myelogenous leukaemia.

It can appear virtually at every body site and during any moment during primary disease evolution. Extramedullary myelogenous leukaemia is defined as being a tumoural lesion composed of immature myeloid cells localised at any extramedullar site. The case of a 39-year old female is reported; she had uterine involvement for this disease. The lesion initially had a clinical appearance of endometrial carcinoma; however, extramedullary myelogenous leukemia with uterine involvement was finally diagnosed in this woman, within an acute myelogenous leukaemia context.

Methodology: Ovid and Medline databases were searched using the following key words: granulocytic sarcoma, extramedullary leukaemia, myeloid sarcoma, female genital tract, uterus, uterine cervix, endometrium. The search was carried out to learn more about this disease, such as frequency, clinical features and diagnosis, treatment and prognosis, according to current medical literature.

Results: two independent reviewers evaluated the papers' relevancy and chose articles dealing with granulocytic sarcoma or mentioning the female genital tract, specifically the uterus (uterus, uterine cervix). A new search was made using references from some papers so found. 123 articles were found referring to different aspects of the disease and the anatomical parts so affected.

14 pertinent papers were chosen, including a review article about extramedullary myeloid leukaemia. Most papers were case reports, brief reports and reviews of some aspects of the disease using small series. No article specifically referring to the endometrium was found.

Conclusion: the uterus is rarely involved in extramedullary myeloid leukaemia. The available information in the medical literature is limited, basically consisting of case reports and analysis of groups from cases reported. There is agreement concerning the difficulty of initial diagnosis, its poor prognosis and treatment, although experience is limited.

Key words: acute myelogenous leukaemia, extramedullary myelogenous leukaemia, granulocytic sarcoma, female genital tract, uterus, endometrium.

\section{REPORTE DE CASO}

Una mujer de 39 años de edad, viuda, G4P3A1, fue remitida en mayo del 2005 con diagnóstico de carcinoma endometrial al Hospital Universitario San Vicente de Paúl de Medellín, Colombia (HUSVP), centro de referencia de tercer nivel. Había consultado a otra institución por menometrorragia y dolor. Allí le realizaron curetaje endometrial y la patología informó: "neoplasia indiferenciada de células pequeñas".

$\mathrm{Al}$ ingreso al HUSVP se encontró una paciente pálida, sin adenopatías o esplenomegalia, sin alteraciones macroscópicas del cérvix. El útero era poco móvil, tenía un tamaño de $12 \mathrm{~cm}$ en su eje longitudinal y se encontró el parametrio izquierdo fijo. El hemoleucograma inicial mostró 11.800 leucocitos con $86 \%$ de blastos, $1 \%$ de neutrófilos y $36 \%$ de linfocitos, hemoglobina de $6,7 \mathrm{~g} / \mathrm{dL}$ y un recuento de plaquetas de 35.000/microlitro. Se encontraron además una deshidrogenasa láctica de 1,977 U/L y una creatinina sérica de 1,5 mg/dL.

La ecografía transvaginal mostró signos de infiltración endometrio-miometrial con útero heterogéneo y aumentado de tamaño. Grosor endometrial de $24 \mathrm{~mm}$. La tomografía axial computarizada reportó hallazgos compatibles con carcinoma endometrial con invasión a la hemipelvis izquierda; topografía cervical mal definida y nódulos pélvicos y retroperitoneales. Además, signos de uropatía obstructiva. 


\section{Estudios de histopatología e inmunohistoquímica}

Se recibió la biopsia de endometrio realizada en el primer hospital, mostró un endometrio comprometido por una neoplasia maligna de aspecto indiferenciado y constituida por células de citoplasma mal definido y núcleo grande, redondo u oval, cromatina vacuolada y nucléolo prominente. La neoplasia infiltraba siguiendo un patrón difuso, respetando las glándulas endometriales (figura 1). Se practicaron estudios de inmunohistoquímica para CD20, CD10, cóctel de citoqueratinas, cromogranina y desmina. Todos fueron negativos.

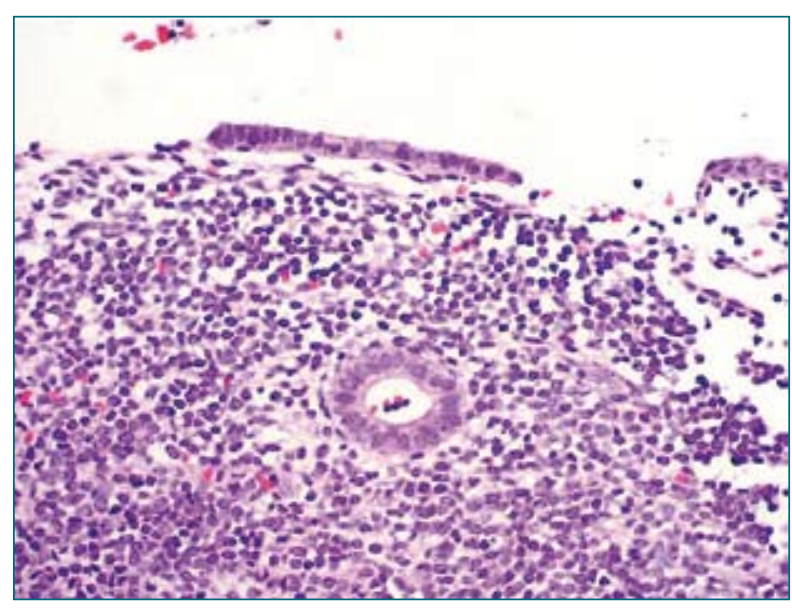

Figura 1. Biopsia de endometrio: compromiso por una neoplasia maligna de aspecto indiferenciado. La neoplasia infiltra siguiendo un patrón difuso, respetando las glándulas endometriales.

Hematoxilina - eosina $200 \mathrm{X}$.

Se recibió también biopsia de médula ósea en la cual se identificó compromiso por una población neoplásica monomorfa semejante a la descrita en la biopsia de endometrio. Se practicaron estudios de inmunohistoquímica (tabla 1 y figura 2) y se hizo el diagnóstico histológico de LMA.

Aunque el recuento total de leucocitos fue normal, en el extendido se evidenció neutropenia y blastos de tamaño medio, con moderada cantidad de citoplasma y escasos gránulos, núcleo con cromatina laxa y nucléolo prominente, algunos tomaban la coloración de peroxidasa.

\begin{tabular}{|c|c|c|}
\hline \multirow{2}{*}{$\begin{array}{l}\text { Marcador } \\
\text { estudiado }\end{array}$} & \multicolumn{2}{|c|}{ Resultado } \\
\hline & Endometrio & Médula ósea \\
\hline CD3 & Negativo & - \\
\hline CD79a & Negativo & Negativo \\
\hline CD 10 & Negativo & - \\
\hline CD 20 & Negativo & - \\
\hline TdT & Negativo & Negativo \\
\hline CD68 & Negativo & Negativo \\
\hline CD56 & Negativo & Negativo \\
\hline CD34 & Positivo & Positivo \\
\hline Mieloperoxidasa & $\begin{array}{c}\text { Focalmente } \\
\text { positivo }\end{array}$ & Positivo \\
\hline
\end{tabular}

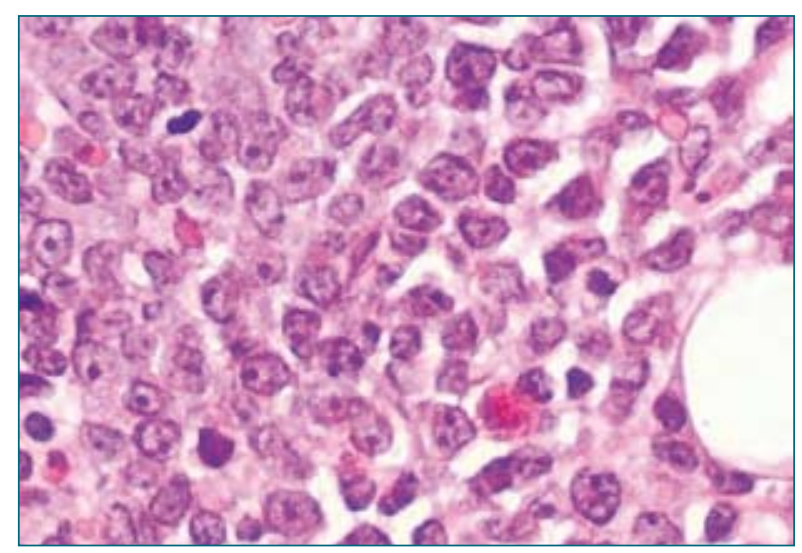

Figura 2. Biopsia de médula ósea. Neoplasia con patrón de crecimiento difuso y células con citoplasma mal definido y núcleo grande, redondo u oval, cromatina vacuolada y nucléolo prominente.

Hematoxilina - eosina $400 \mathrm{X}$.

El estudio citogenético exhibió cariotipo normal 46XX. Con base en la apariencia morfológica y citoquímica de los blastos en la sangre periférica y el aspirado de médula ósea, se diagnosticó LMA M1 de acuerdo con la clasificación del French-.American-British Cooperative Group (FAB) y LMA, con escasa diferenciación según la clasificación de la OMS. Posterior a este diagnóstico, se practicaron estudios de inmunohistoquímica complementarios en endometrio cuyos resultados apoyaron el diagnóstico de compromiso del endometrio por LMA (tabla 1). 
Una vez establecido el diagnóstico de LMA extramedular con compromiso uterino, se realizó un curso de quimioterapia con citarabina (dosis total de $1.000 \mathrm{mg} / \mathrm{m}^{2}$ de superficie corporal) durante los días 1 a 7 más idarubicina (12 mg/m²/día) durante los días 1 a 3 .

La paciente presentó cuadro clínico de neutropenia febril con buena respuesta al manejo médico (según protocolo institucional) que incluyó cobertura antibiótica con ceftriaxona más ciprofloxacina y posteriormente con imipenem y vancomicina. Requirió múltiples transfusiones de hemoderivados (glóbulos rojos empaquetados y concentrado de plaquetas) para el manejo de su anemia y trombocitopenia. La paciente permaneció hospitalizada durante 38 días.

Antes del alta recibió evaluación integral para determinar la respuesta al tratamiento. Se encontró un útero de tamaño normal, móvil y sin lesiones al examen clínico y de ultrasonido transvaginal. La biopsia y el aspirado de médula ósea determinaron la remisión completa de la LMA y la paciente fue dada de alta.

La paciente continuó esquema de quimioterapia de consolidación en otra institución de la ciudad y 14 meses después del diagnóstico se encontraba asintomática.

\section{Revisión de la literatura}

\section{Aspectos históricos}

El sarcoma granulocítico fue descrito por primera vez por Burns en 1811 y en 1853 King lo denominó cloroma debido a la apariencia verdosa del tumor, por el alto contenido de mieloperoxidasa. ${ }^{1}$ No obstante, no todos los tumores exhiben claramente dicha coloración. ${ }^{1,2}$ El término "sarcoma granulocítico" fue usado inicialmente por Rapaport en 1966 para describir una masa tumoral sólida destructiva compuesta de células inmaduras de la serie granulocítica. ${ }^{2,3}$ Posteriormente este término se usó para describir todas las formas de infiltrados leucémicos mieloides extramedulares. ${ }^{3}$ Sin embargo, la denominación de "leucemia mieloide extramedular" da una idea más precisa de su contexto y tiene implicaciones al plantear un tratamiento sistémico para esta enfermedad. ${ }^{1,3}$ En el presente artículo acogemos esta última denominación. Debe resaltarse que Dock y Warthin en 1904 habían dado un paso importante en la comprensión de este tumor al reportar la asociación de este con la leucemia mieloide aguda (LMA). ${ }^{2}$

\section{Epidemiología}

El compromiso del útero por LME es raro y la información disponible en la literatura acerca de esta entidad es escasa. La leucemia mieloide extramedular (LME) ocurre entre el 3 y el 5\% de los pacientes con LMA, según lo reportado en la literatura. ${ }^{3,4}$ Los sitios afectados con más frecuencia son el hueso, los ganglios linfáticos, la piel, el tracto gastrointestinal y la órbita. En la población femenina, el tracto genital y la mama son también afectados aunque con menor frecuencia. ${ }^{1-4}$ El compromiso uterino es cercano al $10 \% \%^{1,5,6}$ y se presenta en un espectro amplio de edades pero más reiterado en la cuarta y quinta décadas de la vida.

Suele haber confusión en el diagnóstico inicial de la LME y se registran altos índices de diagnósticos errados al comienzo del estudio de estas pacientes. ${ }^{4,6,7}$ Se requieren estudios especiales de inmunohistoquímica, citometría de flujo y aun de citogenética, para llegar al diagnóstico correcto y realizar una clasificación adecuada. ${ }^{8,9}$

La quimioterapia sistémica es la piedra angular del tratamiento, pero el pronóstico de esta enfermedad es malo y está relacionado con la evolución de la LMA. ${ }^{2-4,6}$ Se ha descrito la sobrevida entre siete meses y dos años. ${ }^{3,5}$

\section{Presentación clínica}

La presentación clínica de las pacientes con compromiso uterino por LME no es específica e incluye hemorragia uterina anormal en el 82\%, dolor pélvico en el $29 \%$ y síntomas sistémicos en el $17 \%{ }^{5}$ Los hallazgos de una masa en cérvix o de un útero 
agrandado y poco móvil son comunes al examen físico. Se requiere un alto índice de sospecha, en especial cuando aún no hay evidencia de leucemia en la paciente.

Los estudios de imágenes son útiles pero no específicos y en la mayoría de los casos confirman la presencia de una masa ya detectada o sospechada al examen físico. Los estudios histopatológicos e inmunohistoquímicos son necesarios para confirmar el diagnóstico. ${ }^{2,7}$

Se sabe que la LME puede manifestarse antes, durante o después de un cuadro evidente de LMA, en el contexto de la transformación blástica de la leucemia mieloide crónica o en pacientes con síndrome mielodisplásico, sin embargo, tradicionalmente se ha considerado como manifestación de leucemia mieloide aguda. ${ }^{1,7,10}$ Lee y cols. recientemente comunicaron el caso de una paciente en quien la LME del cérvix se presentó como recaída de una LMA tratada dos años atrás y quien había permanecido sin evidencia de la enfermedad. No se documentó reactivación de la LMA y la leucemia mieloide extramedular fue tratada con quimioterapia sistémica, con la cual la paciente alcanzó una buena respuesta clínica. ${ }^{2}$

Cuando la LME es la manifestación inicial, la leucemia mieloide aguda se hará evidente en promedio a los 7,4 meses (intervalo de 8 días hasta 28 meses) luego de la aparición de la lesión extramedular. ${ }^{5}$

\section{Histopatología}

Desde el punto de vista del estudio histopatológico, el compromiso del tracto genital femenino por una LMA es generalmente mal diagnosticado, en particular, cuando el patólogo desconoce la historia clínica. En los casos publicados en la literatura el diagnóstico inicial es correcto en alrededor del $44 \%$ de estos. ${ }^{5}$ Con más frecuencia el diagnóstico inicial es compromiso por linfoma difuso de células grandes, carcinoma indiferenciado, tumores neuroendocrinos, en especial carcinoma neuroendocrino de células grandes, y como sarcomas, particularmente sarcoma estromal endometrial. En muchos casos, solo la inmunohistoquímica permite realizar un correcto diagnóstico diferencial. ${ }^{7}$ Los linfomas difusos B de células grandes casi siempre presentan marcación positiva con CD45 (98\%) y CD20 (97\%). El 66\% son positivos para CD79a y el 41\% para CD10. El tumor mieloide extramedular marca positivamente con mieloperoxidasa en el 88\% de los casos, con CD15 en el 65\%, con CD34 en el $38 \%$ y con CD45 en el 20\%. ${ }^{11,12}$

Se consideran factores de riesgo que predisponen a la presentación extramedular algunas anomalías citogenéticas, marcadores en la superficie celular, la clasificación LMA, M4 y M5 de la FAB con alto recuento de leucocitos al diagnóstico, la disfunción celular inmune, el estrato socioeconómico bajo y el pobre estado nutricional.

Entre las anomalías citogenéticas se destacan la $\mathrm{t}(8 ; 21)$ como la que más se asocia con la $\mathrm{LME},{ }^{12} \mathrm{y}$ la presentación extramedular podría comprometer el buen pronóstico de esta alteración citogenética. ${ }^{13}$ La inv (16) también se presenta con frecuencia e implica buen pronóstico. ${ }^{14}$

La expresión de CD56, CD2, CD4 y CD7 por parte de los blastos, en especial aquellos con diferenciación monocítica o mielomonocítica, se han asociado con una alta incidencia de LME. ${ }^{12}$

\section{Discusión}

El caso que se reporta es llamativo por el compromiso del endometrio, lo cual, desde el punto de vista clínico tiene implicaciones particulares, en especial en lo relacionado con el diagnóstico diferencial. En esta paciente se pensó inicialmente en un carcinoma endometrial. Por otro lado, la edad, cuadro clínico, dificultades en el diagnóstico, características del estudio histopatológico e inmunohistoquímico, complicaciones de la enfermedad y el tratamiento recibido son similares a lo informado en la literatura. ${ }^{5}$

Debido a que las pacientes con LME del tracto genital en un contexto de enfermedad primaria (LMA) aún no diagnosticada, por lo general, se presentan con síntomas que son comunes a otras entidades, como cáncer primario del endometrio o 
del cérvix, o a causas benignas de sangrado genital o dolor pélvico, el diagnóstico rara vez se plantea durante la evaluación inicial, pero finalmente, es necesario para la realización de un tratamiento correcto y oportuno.

Por otro lado, el hallazgo de una masa tumoral en el tracto genital de una paciente con LMA ya diagnosticada, hace necesario que se establezca con claridad si se trata de una LME o de otro tipo de enfermedad maligna o benigna, lo cual tiene implicaciones para el tratamiento y requiere un esfuerzo diagnóstico por un equipo médico altamente especializado.

\section{REFERENCIAS}

1. Byrd JC, Edenfield WJ, Shields DJ, Dawson NA. Extramedullary myeloid cell tumors in acute nonlymphocytic leukemia: a clinical review. J Clin Oncol 1995;13:1800-16.

2. Lee JW, Kim YT, Min YH, Kim JW, Kim SH, Park $\mathrm{KH}$, et al. Granulocytic sarcoma of the uterine cervix. Int J Gynecol Cancer 2004;14:553-7.

3. Breccia M, Mandelli F, Petti MC, D`Andrea M, Pescarmona E, Pileri SA, et al. Clinico-pathological characteristics of myeloid sarcoma at diagnosis and during follow-up: report of 12 cases from a single institution. Leuk Res 2004;28:1165-9.

4. Friedman HD, Adelson MD, Elder RC, Lemke SM. Granulocytic sarcoma of the uterine cervix - literature review of granulocytic sarcoma of the female genital tract. Gynecol Oncol 1992;46:128-37.

5. Pathak B, Bruchim I, Brisson ML, Hammouda W, Bloom C, Gotlieb WH. Granulocytic sarcoma presenting as tumors of the cervix. Gynecol Oncol 2005;98:493-7.

6. Oliva E, Ferry JA, Young RH, Prat J, Srigley JR, Scully RE. Granulocytic sarcoma of the female genital tract: a clinicopathologic study of 11 cases. Am J Surg Pathol 1997;21:1156-65.

7. Menasce LP, Banerjee SS, Beckett E, Harris M. Extra-medullary myeloid tumour (granulocytic sarcoma) is often misdiagnosed: a study of 26 cases. Histopathology 1999;34:391-8.

8. Bennett JM, Catovsky D, Daniel MT, Flandrin G, Galton DA, Gralnick HR, et al. Proposals for the classification of the acute leukaemias. FrenchAmerican-British (FAB) cooperative group. Br J Haematol 1976;33:451-8.

9. Jaffe ES, Harris NL, Stein H, Vardiman JW, eds. World Health Organization classification of tumours. Pathology and genetics of tumours of haematopoietic and lymphoid tissues. Lyon, France: IARC Press;2001.

10. Chang CC, Eshoa C, Kampalath B, Shidham VB, Perkins S. Immunophenotypic profile of myeloid cells in granulocytic sarcoma by immunohistochemistry. Correlation with blast differentiation in bone marrow. Am J Clin Pathol 2000;114:807-11.

11. Audouin J, Comperat E, Le Tourneau A, CamilleriBroët S, Adida C, Molina T, et al. Myeloid sarcoma: clinical and morphologic criteria useful for diagnosis. Int J Surg Pathol 2003;11:271-82.

12. Tallman MS, Hakimian D, Shaw JM, Lissner GS, Russell EJ, Variakojis D. Granulocytic sarcoma is associated with the 8;21 translocation in acute myeloid leukemia. J Clin Oncol 1993;11:690-7.

13. Byrd JC, Weiss RB, Arthur DC, Lawrence D, Baer MR, Davey F, et al. Extramedullary leukaemia adversely affects hematologic complete remission rate and overall survival in patients with $\mathrm{t}(8 ; 21)(\mathrm{q} 22 ; \mathrm{q} 22)$ : results from Cancer and Leukemia Group B 8461. J Clin Oncol 1997;15:466-75.

14. Holmes R, Keating MJ, Cork A, Broach Y, Trujillo J, Dalton WT Jr, et al. A unique pattern of central nervous system leukemia in acute myelomonocytic leukemia associated with inv(16)(p13q22). Blood 1985;65:1071-8. 